\title{
Saturated fatty acid-induced cardiomyopathy with diastolic dysfunction can be ameliorated by changing the quality of fatty acids to monounsaturated fatty acid
}

\author{
Type
}

Research paper

\section{Keywords}

diastolic dysfunction, SIRT1, saturated fatty acids, monounsaturated fatty acids, stearoyl-CoA desaturase-1, membrane fatty acid composition

\begin{abstract}
Introduction

Lipotoxicity due to obesity is known to lead to cardiac dysfunction. In an earlier study, we found that an increase in the ratio of saturated fatty acids (SFA) to monounsaturated fatty acids (MUFA) in the membrane of cardiomyocytes causes endoplasmic reticulum (ER) stress. Such stress is hypothesized to be involved in development of SFA-related cardiomyopathy. Another factor affecting the membrane SFA/MUFA ratio is suppression by SFA of SIRT1-mediated stearoyl-CoA desaturase-1 (SCD1), which is involved in converting SFA to MUFA. Therefore, we evaluated whether increasing dietary intake of MUFA can improve the membrane SFA/MUFA ratio.
\end{abstract}

Material and methods

Wild-type mice $(n=30)$ and cardiomyocyte-specific SIRT1 knockout mice $(n=30)$ were randomly divided into 3 groups and assigned to 16 weeks of a standard mouse diet, 16 weeks of an SFA-rich high-lard diet (HLD), or 8 weeks of a HLD followed by 8 weeks of a MUFA-rich high olive oil diet (HOD switch).

\section{Results}

Compared with the control group, the wild-type mice on the HOD diet showed normalized SIRT1-mediated SCD1 signaling, increased membrane SFA/MUFA ratio, decreased ER stress, and improved cardiomyopathy variables. The HLD-fed SIRT1 knockout mice showed greater changes in the SFA/MUFA ratio, ER stress, and cardiomyopathy variables than the wild-type mice. Switching from HLD to HOD ameliorated these phenotypes, although it did not restore the reduced expression of SCD1.

\section{Conclusions}

The MUFA-rich diet was found to have a therapeutic effect on SFA-induced cardiomyopathy with diastolic dysfunction by directly rebalancing membrane fatty acid oversaturation and indirectly through the de-inhibition of SIRT1/SCD1 signaling. 
Saturated fatty acid-induced cardiomyopathy with diastolic dysfunction can be ameliorated by changing the quality of fatty acids to monounsaturated fatty acid

Tsunehisa Yamamoto, MD, $\mathrm{PhD}^{1}$; Jin Endo, MD, $\mathrm{PhD}^{1,2}$; Masaharu Kataoka, $\mathrm{MD}, \mathrm{PhD}^{1}$;

Yoshinori Katsumata, MD, $\mathrm{PhD}^{1}$; Kohsuke Shirakawa, MD, $\mathrm{PhD}^{1}$; Sarasa Isobe, $\mathrm{MD}^{1}$;

Hidenori Moriyama, MD¹; Shinichi Goto, MD, PhD'; Yuta Shimanaka, PhD³; Nozomu Kono, $\mathrm{PhD}^{3}$; Ken Shinmura, MD, $\mathrm{PhD}^{1,4}$; Hiroyuki Arai, $\mathrm{PhD}^{3}$; Keiichi Fukuda, MD, $\mathrm{PhD}^{1}$; Motoaki Sano, MD, $\mathrm{PhD}^{1,2 *}$

${ }^{1}$ Department of Cardiology, Keio University School of Medicine, Tokyo, Japan

${ }^{2}$ Japan Science and Technology Agency, Tokyo, Japan

${ }^{3}$ Graduate School of Pharmaceutical Sciences, Tokyo University, Tokyo, Japan

${ }^{4}$ Department of General Medicine, Hyogo College of Medicine, Hyogo, Japan

*Address for correspondence: Dr. Motoaki Sano, Department of Cardiology, Keio University School of Medicine, 35 Shinanomachi, Shinjuku-ku, Tokyo 160-8582, Japan. Tel:+81-(3)-5363-3874, Fax: +81-(3)-5363-3875; E-mail: msano@a8.keio.jp 


\section{Abstract}

$<$ Introduction > Lipotoxicity due to obesity is known to lead to cardiac dysfunction. In an earlier study, we found that an increase in the ratio of saturated fatty acids (SFA) to monounsaturated fatty acids (MUFA) in the membrane of cardiomyocytes causes endoplasmic reticulum (ER) stress. Such stress is hypothesized to be involved in development of SFA-related cardiomyopathy. Another factor affecting the membrane SFA/MUFA ratio is suppression by SFA of SIRT1-mediated stearoyl-CoA desaturase-1 (SCD1), which is involved in converting SFA to MUFA. Therefore, we evaluated whether increasing dietary intake of MUFA can improve the membrane SFA/MUFA ratio.

$<$ Material and Methods> Wild-type mice $(n=30)$ and cardiomyocyte-specific SIRT1 knockout mice $(n=30)$ were randomly divided into 3 groups and assigned to 16 weeks of a standard mouse diet, 16 weeks of an SFA-rich high-lard diet (HLD), or 8 weeks of a HLD followed by 8 weeks of a MUFA-rich high olive oil diet (HOD switch).

$<$ Results > Compared with the HLD group, the wild-type mice on the HOD switch showed normalized SIRT1-mediated SCD1 signaling, membrane SFA/MUFA ratio, decreased ER stress, and improved cardiomyopathy variables. The HLD-fed SIRT1 knockout mice showed greater changes in the SFA/MUFA ratio, ER stress, and cardiomyopathy 
variables than the wild-type mice. Switching from HLD to HOD ameliorated these phenotypes, although it did not restore the reduced expression of SCD1.

$<$ Conclusions $>$ The MUFA-rich diet was found to have a therapeutic effect on SFAinduced cardiomyopathy with diastolic dysfunction by directly rebalancing membrane fatty acid oversaturation and indirectly through the de-inhibition of SIRT1/SCD1 signaling in mice.

Keywords: saturated fatty acids, monounsaturated fatty acids, diastolic dysfunction, SIRT1, stearoyl-CoA desaturase-1, membrane fatty acid composition 


\section{Introduction}

Obesity become world-wide health problem [1, 2]. Dyslipidemia is caused by overeating, lack of exercise, and aging and results in higher fatty acid uptake by cells. In the hearts of people with diabetes, the imbalance between cell fatty acid levels and mitochondrial $\beta$-oxidation of fatty acid was found to cause cardiac dysfunction. The associated mechanisms include a build-up of intermediates of fatty acid metabolism in cardiomyocytes and an increase in harmful reactive oxygen species [3].

Our research has focused on the effect on cardiac function of not only the quantity but also the quality of fatty acid taken up by cardiomyocytes. In our earlier work, we showed that both mice on a high-lard diet (HLD), which has high levels of saturated fatty acids (SFA), and those on a high olive oil diet (HOD), which has high levels of monounsaturated fatty acids (MUFA), became obese; however, left ventricular (LV) diastolic dysfunction was more severe in the former group [4]. This difference was not due to differences in the degree of mitochondrial $\beta$-oxidation activity or to differences in the amount of toxic lipid intermediates accumulated in cardiomyocytes. Rather, LV diastolic dysfunction was found to correlate with both the presence of endoplasmic reticulum (ER) stress and an increase in the ratio of SFA to MUFA in phospholipids in the membrane of the ER. Organelle function, including that of the ER, depends on 
phospholipid homeostasis in membranes [5]. An increase in the SFA/MUFA ratio in the ER membrane causes it to stiffen, which leads to an activation of transmembrane protein kinase RNA-like endoplasmic reticulum kinase (PERK) and inositol requiring enzyme-1 (IRE1) independently of the accumulation of misfolded proteins in the ER lumen [6]. Protein stearoyl-CoA desaturase-1 (SCD1), a catalyst of a rate-limiting step in the production of unsaturated fatty acids in the ER membrane, also regulates the ratio of SFA to MUFA in phospholipids [7]. Phosphorylation of adenosine monophosphateactivated protein kinase (AMPK) was found to be decreased in hearts of mice fed an HLD, leading to reduced expression of SIRT1, which is known to mediate the changes in transcription that result from reduced calorie intake and increased exercise [8]. Moreover, transcriptional activity of the liver X receptors (LXRs) is selectively repressed by reduced expression of SIRT1. Since the expression of SCD1 is regulated by LXRs, SCD1 expression is suppressed in the HLD-fed heart, resulting in impaired conversion of SFA to MUFA in the ER membrane. In contrast, phosphorylation of AMPK and SIRT1 expression were not suppressed and SCD1 expression was maintained in the hearts of HOD-fed mice. Furthermore, we have previously shown that HLD-induced LV diastolic dysfunction can be exacerbated by cardiomyocyte-specific SIRT1 knockout、 ameliorated by either nicotinamide mononucleotide (NMN)-induced activation of SIRT1 or an LXR 
agonist in mice [9]. The mechanisms by which ER membrane stiffening impairs diastolic function include decreased $\mathrm{Ca}^{2+}$ pumping to the sarcoplasmic reticulum [10], pathological cardiac hypertrophy due to increased expression of transient receptor potential channels (TRPC) [11], and cardiomyocyte death due to activation of the X-box binding protein 1 (XBP1)-independent IRE1 pathway [12].

In this study, we hypothesized that switching from HLD to HOD could reverse the ER membrane stiffening and cardiomyopathy with diastolic dysfunction induced by HLD. If this hypothesis is confirmed, a MUFA-rich diet may represent a novel treatment for SFArelated cardiomyopathy.

\section{Material and Methods}

Animal studies: We crossed SIRT1 flox/flox mice, which have a C57BL/6J background, with a-myosin heavy chain promoter-driven Cre mice (aMHC-Cre) to create cardiomyocyte-specific SIRT1 knockout mice (SIRT1CKO), as previously described [13], and then backcrossed these mice to the C57BL/6J background. Mice were kept in a 12:12 light-dark cycle and provided with food ad libitum; food consisted of a standard mouse diet (SD; D12450J, 10kcal\% fat, Research Diets, see Online Table 1), an HLD (D12492, 60kcal\% fat, Research Diets, see Online Table 1), or an HOD (D01112603, 
$60 \mathrm{kcal} \%$ fat, Research Diets, see Online Table 1). All diets were purchased from Research Diets, Inc. Fat accounted for $60 \%$ of the total calories in HLD and HOD and only $10 \%$ of total calories in SD. HLD and HOD consequently had more calories per gram than SD. All three diets provided the same amounts for sucrose, minerals, and vitamins for the same calorie intake. Lard indicates porcine origin fat as opposed to beef or lamb fat origin. Though all will be rich in saturated fat, porcine lard can be higher in monounsaturated fat compared to beef or sheep.

Glucose and insulin tolerance tests: We performed glucose tolerance (oral administration of $1.5 \mathrm{~g} / \mathrm{kg}$ of glucose, after $16 \mathrm{~h}$ of fasting) and insulin tolerance (administration of $0.75-2 \mathrm{U} / \mathrm{kg}$ of insulin intraperitoneally, after $4 \mathrm{~h}$ of fasting) tests to assess glucose intolerance and insulin resistance as described [14].

Assessment of fatty acids in phospholipids: We extracted lipids from heart tissue according to the method published by Bligh and Dyer [15], as described in an earlier publication [4]. In brief, we applied the lipid extract to a silica column with aminopropyl groups (InertSep ${ }^{\circledR} \mathrm{NH} 2$, GL sciences, Tokyo, Japan) to obtain isolated phospholipids, which we then methylated with $2.5 \% \mathrm{H}_{2} \mathrm{SO}_{4}$ in methanol. This process generated fatty acid methyl esters, which we extracted with hexane and analyzed by gas chromatography-mass spectrometry (GC-MS) with an Agilent 7890A-5975C GC-MS 
network system (Agilent Technologies, Wilmington, DE, USA) with a DB-23 capillary column $(60 \mathrm{~nm} \times 250 \mu \mathrm{L} \times 0.15 \mu \mathrm{m}$; Agilent Technologies). Initially, the temperature in the GC-MS network system was kept at $50^{\circ} \mathrm{C}$ for 1 minute. Then, it was increased by $25^{\circ} \mathrm{C}$ per minute to $175^{\circ} \mathrm{C}$ and then by $5^{\circ} \mathrm{C}$ per minute to $235^{\circ} \mathrm{C}$ and kept at this temperature for 5 minutes. Both the injector and the detector were kept at a temperature of $250^{\circ} \mathrm{C}$.

Measurements of hemodynamic pressure-volume: We measured hemodynamic pressure-volume with a pressure-conductance catheter, in accordance with a previously described method [4]. In brief, after anesthetizing the mice with isoflurane, we inserted a 1.4F pressure-conductance catheter (SPR-839; Millar instruments) into the right carotid artery. First, the catheter was advanced as far as the ascending aorta to enable measurement of aortic blood pressure. Then, the catheter was further advanced into the left ventricle, where it was left in place to enable continuous measurement of pressurevolume (PV) signals with a Mikro-Tip® Pressure Volume System (MRVS Ultra, Millar instruments); digital PV signals were acquired with MPVS PL3508 (PowerLab 8/35, ADInstruments) at $2 \mathrm{kHz}$ and analyzed offline with LabChart 8 pro (ADInstruments). Five minutes after insertion of the catheter into the left ventricle, baseline measurements of LV end-systolic pressure (LVESP) and end-diastolic pressure (LVEDP) and cardiac output (CO) were recorded for 10 consecutive beats. Subsequently, we performed 
transient occlusion of the inferior vena cava and measured end-systolic elastance (Ees), end-systolic volume (ESV), end-diastolic volume (EDV), and $\mathrm{V}_{0}$ over multiple beats. We used these values to calculate the linear LVESP-volume relationship (ESPVR), a measure of cardiac contractility, with the following equation: ESPVR $=$ Ees $\times E S V+V_{0}$ In addition, we calculated the exponential LVEDP-volume relationship (EDPVR), a measure of end-diastolic stiffness, as follows: EDPVR $=\alpha \times \exp ^{\beta \times E D V}$.

Echocardiography: We performed short-axis echocardiography with the Vevo 660 system (Visual Sonics) in accordance with $[4,16]$ in 20-week-old mice after anesthetizing them by using inhaled isoflurane (1.5\%) and anchoring them in a supine position. We assessed LV end-systolic diameter (LVESD) and LV end-diastolic diameter (LVEDD) in accordance with the leading-edge convention of the American Society of Echocardiography. The, we calculated the percentage of LV fractional shortening (FS) as ([LVEDD-LVESD]/LVEDD) × 100

Histology: We performed the steps for analyzing histology as described in a previous publication [4]. In brief, we anesthetized and then euthanized the mice. After perfusing hearts with phosphate-buffered serum, we fixed them with $10 \%$ formalin neutral buffer solution and embedded them in paraffin. We then stained them with hematoxylin \& eosin, Azan blue, Oil red $\mathrm{O}$ and wheat germ agglutinin. The fibrotic area and Oil red $\mathrm{O}$ staining 
area were quantitatively assessed by staining short-axis sections in the mid-level of the left ventricle with Azan blue or Oil red $\mathrm{O}$ and comparing staining with control reference sections with BZ-II Analyzer software (Keyence) together with a BIOREVO BZ-9000 microscope (Keyence). The software counts the number of pixels in each section that have a prespecified amount of staining and calculates the corresponding area. We also used images from sections stained by wheat germ agglutin to assess the cross-sectional area of myocytes by tracing cardiomyocyte outlines in each section with ImageJ software. Apoptosis: We evaluated apoptotic fragmentation of DNA by terminal deoxynucleotidyl transferase dUTP nick end labeling (TUNEL) and changes in nuclear morphology by DAPI staining, as described elsewhere [4]. To quantify apoptosis, we manually counted the TUNEL-positive and DAPI-stained nuclei in 8 to 10 fields per sample.

Western blotting: We performed western blotting to evaluate AMPK phosphorylation and SCD1, SIRT1, and glyceraldehyde 3-phosphate dehydrogenase (GAPDH) expression, as described in [4]. To do so, we extracted the total protein from frozen hearts and livers from mice and studied them by standard SDS-PAGE. We used the following primary antibodies: anti-phospho-AMPKa (Thr172; \#4188), anti-AMPK (\#2532), anti-SIRT1 (\#8649), anti-SCD1 (\#2794), and anti-GAPDH (\#2118) (Cell Signaling Technology, Danvers, MA, USA). 
Quantitative real-time polymerase chain reaction: We used quantitative real-time polymerase chain reaction (PCR) to determine levels of RNA in samples from hearts, as described in our previous work [4]. Samples were first treated with Trizol reagent (Invitrogen), and then a PCR template of cDNA was obtained by reverse transcribing the total RNA (0.1-0.3 $\mu \mathrm{g})$ with an RNA PCR kit (Takara Biotechnology, Otsu, Shiga, Japan). The template was used to assess RNA levels by real-time PCR with the ABI PRISM7700 Sequence Detector (Applied Biosystems). To calculate the relative expression levels of the target genes, we used the comparative cycle threshold (Ct) method and normalized target gene amounts by comparing them with amplified Gapdh (an internal control). The primer sequences of the target genes can be found in Online Table 2.

Quantitative real-time PCR for microRNA: Expression levels of microRNA(miR)-195 and miR-451 were measured as described in our previous work [4]

Statistical analysis: Data are shown as mean (SEM). Differences among multiple groups were compared using ANOVA followed by post hoc Tukey-Kramer tests. A normality test was performed before applying multiple group statistical comparison. The statistical significance level was set at $P<0.05$.

Ethical considerations: The International Animal Care and Use Committee of Keio University School of Medicine approved the study (12063). All study procedures were 
performed in accordance with the principles described in the US National Institutes of Health guide for the care and use of laboratory animals.

\section{Results}

\subsection{Switching from SFA-rich diet to MUFA-rich diet reduced SFA-induced cardiomyopathy with diastolic dysfunction}

4-week-old wild-type male mice were randomly assigned to 16 weeks of SD, HLD, or an 8 weeks of HLD followed by a switch to 8 weeks of HOD (HOD switch; Figure 1A). At 20 weeks of age, we found no differences in body weight or visceral fat weight between the HLD and HOD switch groups although HLD group developed a more severe glucose intolerance and insulin resistance than HOD switch group (Online Figure 1A-C). The HOD switch group showed less severe cardiac hypertrophy (i.e., they showed a smaller increase in the ratio of heart weight to tibia length and in cell surface area), fibrosis, and cardiomyocyte apoptosis (Figure 1B-E), along with diminished induction of cardiac stress, fibrosis response markers and TRPC (Myh7, Tgfb1, Col1a1, and Trpc 1/3/6; Figure 1F, Online Figure 1D) compared to 16 weeks of HLD. There was no difference in lipid accumulation between the HLD and HOD switch groups (Online Figure 1E). LV diastolic dysfunction, which was estimated from LVEDP and the EDPVR slope, was also improved 
by HOD switch (Figure 1G-H, Online Table 3) compared to 16 weeks of HLD. Because end-systolic pressure (ESP) was not different between the HLD and the HOD switch group, the improvement in LV diastolic function by HOD switch was thought to be due to changes in the myocardium itself, not to afterload reduction (Online Table 3).

\subsection{HLD-induced increase in the membrane SFA/MUFA ratio and ER stress were} ameliorated by HOD switch in heart

After 16 weeks of HLD, the proportion of stearate (C18:0) had increased and that of oleate (C18:1) had decreased, resulting in an increase in the ratio of SFA to MUFA in membrane phospholipids. The HOD switch corrected this HLD-induced oversaturation of membrane phospholipids (Figure 2A). Consistent with rebalancing of membrane SFA/MUFA ratio, HLD-induced ER stress was reduced by HOD switch compared to 16 weeks of HLD (Figure 2B). Furthermore, HOD switch restored the phosphorylation of AMPK, expression of SIRT1, and target genes of LXRs such as Scd1, which had been suppressed by HLD (Figure 2C-E). Compared to HLD groups, HOD switch group showed mild induction of microRNA (miR)-451 and miR-195 (Online Figure 1F), which play as suppressors of AMPK and SIRT1 respectively $[17,18]$. On the other hand, HOD switch did not affect the expression of transcriptional regulators involved in mitochondria 
biogenesis such as Pgc1/Tfam/Nrf1, triglyceride turnover-related genes such as Pnpla2,

Dgat1, target genes of peroxisome proliferator-activated receptor alpha (PPAR $\alpha)$ involved in fatty acid $\beta$-oxidation such as Cpt1b, Cd36, Acs/1, and target genes of forkhead box protein 01 (FoxO1) involved in antioxidant stress response such as Sod2, Catalase, all of which were upregulated by HLD (Figure 2F).

\subsection{HOD switching had a protective effect against HLD-induced cardiomyopathy in SIRT1 knockout heart}

Previously, we showed that deletion of cardiomyocyte-specific SIRT1 showed vulnerability to HLD-induced cardiomyopathy and membrane fatty acid saturation [9]. Therefore, we next tested the therapeutic effect of switching from HLD to HOD in cardiomyocyte-specific SIRT1 knockout mice (SIRT1CKO; Figure 3A, Online Figure 2A). At 20 weeks of age, body weight and visceral fat weight were not different between the HLD and HOD switch groups (Online Figure 3B-C). HOD switch reduced pathological hypertrophy, fibrosis, cardiomyocyte death, and LV diastolic dysfunction (increased LVEDP and EDPVR) in SIRT1CKO compared to 16 weeks of HLD, but these effects were less drastic in SIRT1CKO than in wild-type (Figure 3B-H, Online Figure 2D, Online Table 4). 


\subsection{In SIRT1CKO mice, HOD switching ameliorated the HLD-induced increase in the membrane SFA/MUFA ratio and ER stress}

The effect of HOD switch on reducing the membrane SFA/MUFA ratio and the concomitant attenuation of ER stress was less pronounced in SIRT1CKO than in wildtype mice (Figure 4A-B). Notably, SIRT1CKO hearts did not show the recovery from the HLD-induced decrease in SCD1 expression and target genes of LXRs after switching to HOD (Figure 4C-D). HOD switch did not affect the expression of transcriptional regulators involved in mitochondria biogenesis, triglyceride turnover-related genes, target genes of PPARa, and target genes of FoxO1, all of which were upregulated by HLD (Figure 4E).

\section{Discussion}

This study showed that the increase in the ratio of SFA to MUFA in the membrane induced by an HLD in mice and the associated pathological cardiac hypertrophic remodeling with impaired LV diastolic function can be corrected by switching from HLD to HOD. Part of the effect of switching to HOD was the release of HLD-induced suppression of SIRT1 activity, which proved that restoring SCD1 expression is an important step in maintaining the membrane SFA/MUFA ratio by converting SFA to MUFA 
locally in the ER membrane. However, HOD switch also improved membrane SFA/MUFA ratio even in SIRT1CKO hearts. This result suggests that HOD switch can directly correct membrane fatty acid composition independent of SIRT1/SCD1 signaling (Figure 4F).

Oleate is known as a most preferentially utilized fatty acids in hearts [19]. The benefits of increasing the intake of unsaturated fatty acids (UFA) as an alternative to dietary SFA have been shown by the results of clinical studies. One study found that if patients with heart failure with preserved ejection fraction (HFpEF) consume foods rich in UFA, which consist of MUFA and polyunsaturated fatty acid (PUFA), both their cardiac diastolic function and cardiorespiratory fitness improve [20]. Furthermore, based on these results, HFpEF patients were instructed to actively consume UFA for 12 weeks, which improved their cardiorespiratory fitness [21]. However, it was unclear whether MUFA or PUFA showed more efficiency against HFpEF. Our results suggest that MUFA-rich diet can prevent the progression of SFA-induced cardiomyopathy. Increasing MUFA intake may have a beneficial effect for hearts with low SIRT1 activity, such as aged hearts [22].

Overall, there is conflicting and limited evidence about the links between serum MUFAs and atherosclerotic cardiovascular disease (CVD) $[23,24,25,26]$. In this study, we showed that myocardial diastolic dysfunction progressed with an SFA-rich diet and that switching from an SFA-rich diet to a MUFA (oleate)-rich diet improved diastolic 
dysfunction. We found a strong correlation between the severity of diastolic dysfunction and the membrane SFA/MUFA ratio in the myocardium. The etiology and pathophysiology of heart failure in the general population are changing dramatically because of lifestyle changes and the aging of the population. We believe that this study has raised the possibility that MUFA (oleate) may be effective in the treatment of nonischemic HFpEF related to obesity. In the present study, we analyzed a simplified mouse model of HFpEF caused by a diet high in SFA. In clinical practice, the etiology of HFpEF is more multifaceted and more complex. Also, in patients with HFpEF we should consider that the contribution of an increased membrane SFA/MUFA ratio to diastolic dysfunction varies from patient to patient. Whether switching from an SFA-rich diet to a MUFA (oleate)-rich diet is effective in preventing or treating HFpEF in the real world needs to be tested in future clinical interventional trials.

We previously analyzed hearts in 20-week-old mice after they were fed a HLD from the age of 4 weeks $[4,9]$. In this study, we tested the significance of changing from HLD to $\mathrm{HOD}$ at an intermediate age of 12 weeks. Other research groups have reported that 8 weeks of HLD loading is sufficient to induce LV hypertrophy, fibrosis, and diastolic dysfunction [27]. A limitation of the present study is that we did not assess the heart at the time of changing from HLD to HOD. Therefore, we could not determine whether the 
change in HOD reversed the hypertrophy, fibrosis, and diastolic dysfunction of the left ventricle formed by HLD or stopped the deterioration of the condition after 8 weeks of age. In any case, we demonstrated that early conversion from HLD to HOD was effective in preventing and treating disease progression in the HLD-loaded HFpEF mouse model.

\section{Conclusions}

we demonstrate that MUFA-rich diet counteracted SFA-induced cardiomyopathy with diastolic dysfunction. As a part of the mechanism, we identified that MUFA-rich diet prevented the SFA-induced increased in membrane SFA/MUFA ratio via SIRT1/SCD1 dependent and independent pathways.

\section{Acknowledgments}

We thank Yoshiko Miyake (Keio University, Japan) for technical assistance.

\section{Funding}

This study was supported by JST PRESTO grants (2013-2015), JSPS KAKENHI grants (15H04825 and 15H01160; to Dr. Sano) and by Takeda Science Foundation grants, JSPS KAKENHI grants (16K21358; to Dr. Yamamoto). 


\section{References}

[1] Jana K, Ewelina W, Alicja Z. Obesity. An analysis of epidemiological and prognostic research. Arch Med Sci 2015; 11(1): 24-33.

[2] Jun Z, Xiaohua S, Gang L, et al. The incidence of acute myocardial infarction in relation to overweight and obesity: a meta-analysis. Arch Med Sci 2014; 10(5): 855-62.

[3] Michinari N, Junichi S. Cardiomyopathy in obesity, insulin resistance and diabetes. J Physiol 2020; 598(14): 2977-2993.

[4] Tsunehisa Y, Jin E, Masaharu K, et al. Decrease in membrane phospholipid unsaturation correlates with myocardial diastolic dysfunction. PLoS One 2018; 13(12): e0208396.

[5] Nurulain H, Chengchao X, Guillaume T. From the unfolded protein response to metabolic diseases - lipids under the spotlight. J Cell Sci 2018; 131(3): jcs199307.

[6] Kristina H, Kristina P, Roberto C, et al. Activation of the Unfolded Protein Response by Lipid Bilayer Stress. Mol Cell 2017;67(4):673-684.e8.

[7] Ariyama H, Kono N, Matsuda S, Inoue T, Arai H. Decrease in membrane phospholipid unsaturation induces unfolded protein response. J Biol Chem 2010; 285(29): 2202722035. 
[8] Tsunehisa Y, Ken S. Chapter 17: Targeting sirtuins to modulate energy metabolism in heart disease. Sirtuin Biology in Medicine. (eds. Maiese K) Academic Press Elsevier; 2021. p. 285-293.

[9] Tsunehisa Y, Jin E, Masaharu K, et al. Sirt1 counteracts decrease in membrane phospholipid unsaturation and diastolic dysfunction during saturated fatty acid overload. J Mol Cell Cardiol 2019; 133: 1-11.

[10] Suneng F, Ling Y, Ping L, et al. Aberrant lipid metabolism disrupts calcium homeostasis causing liver endoplasmic reticulum stress in obesity. Nature 2011; 473(7348): 528-31.

[11] Haodi W, Huaxiao Y, June-Wha R, et al. Modelling diastolic dysfunction in induced pluripotent stem cell-derived cardiomyocytes from hypertrophic cardiomyopathy patients. Eur Heart J 2019; 40(45): 3685-3695.

[12] Tsunehisa Y, Jin E, Masaharu K, et al. Palmitate induces cardiomyocyte death via inositol requiring enzyme-1 (IRE1)-mediated signaling independent of X-box binding protein 1 (XBP1). Biochem Biophys Res Commun 2020; 526(1): 122-127.

[13] Yamamoto T, Tamaki K, Shirakawa K, et al. Cardiac Sirt1 mediates the cardioprotective effect of caloric restriction by suppressing local complement system 
activation after ischemia-reperfusion. Am J Physiol Heart Circ Physiol 2016; 310: H1003-H1014.

[14] Shirakawa K, Yan X, Shinmura K, et al. Obesity accelerates T cell senescence in murine visceral adipose tissue. J Clin Invest 2016;126(12):4626-39.

[15] Bligh EG, Dyer WJ. A rapid method of total lipid extraction and purification. Can J Biochem Physiol 1959; 37(8): 911-917.

[16] Matsuhashi T, Hishiki T, Zhou H, et al. Activation of pyruvate dehydrogenase by dichloroacetate has the potential to induce epigenetic remodeling in the heart. $\mathrm{J} \mathrm{Mol} \mathrm{Cell}$ Cardiol 2015; 82: 116-124.

[17] Zhu H, Yang Y, Wang Y, et al. MicroRNA-195 promotes palmitate-induced apoptosis in cardiomyocytes by down-regulating Sirt1. Cardiovasc Res 2011; 92: 75-84.

[18] Kuwabara Y, Horie T, Baba O, et al. MicroRNA-451 exacerbates lipotoxicity in cardiac myocytes and high-fat diet-induced cardiac hypertrophy in mice through suppression of the LKB1/AMPK pathway. Circ Res 2015; 116: 279-288.

[19] Danielle M, Cholsoon J, Michael N, et al. Comprehensive quantification of fuel use by the failing and nonfailing human heart. Science 2020; 370(6514): 364-368. 
[20] Salvatore C, Justin M. C, Leo F. B, et al. Dietary Fat, Sugar Consumption, and Cardiorespiratory Fitness in Patients With Heart Failure With Preserved Ejection Fraction. JACC Basic TransI Sci 2017; 2(5): 513-525.

[21] Salvatore C, Hayley E. B, Justin M. C, et al. Unsaturated Fatty Acids to Improve Cardiorespiratory Fitness in Patients With Obesity and HFpEF: The UFA-Preserved Pilot Study. JACC Basic TransI Sci 2019; 4(4): 563-565.

[22] Sarah C, Francesco P, Francesco C. Ageing, metabolism and cardiovascular disease. J Physiol 2016; 594(8): 2061-73.

[23] Mazidi M, Mikhailidis DP, Sattar N, et al. Association of types of dietary fats and allcause and cause-specific mortality: A prospective cohort study and meta-analysis of prospective studies with 1,164,029 participants. Clin Nutr. 2020;39(12):3677-3686.

[24] Mazidi M, Katsiki N, Shekoohi N, Banach M. Monounsaturated Fatty Acid Levels May Not Affect Cardiovascular Events: Results From a Mendelian Randomization Analysis. Front Nutr 2020; 7:123.

[25] Cicero AFG, Colletti A, von Haehling S, et al. International Lipid Expert Panel. Nutraceutical support in heart failure: a position paper of the International Lipid Expert Panel (ILEP). Nutr Res Rev 2020; 33(1):155-179. 
[26] Pan Z, Yu Z, Wei H, et al. Dietary Fats in Relation to Total and Cause-Specific Mortality in a Prospective Cohort of 521120 Individuals With 16 Years of Follow-Up. Circ Res 2019; 124(5): 757-768.

[27] Mingming T, Toshiro S, Peiyong Z, et al. Mitophagy Is Essential for Maintaining Cardiac Function During High Fat Diet-Induced Diabetic Cardiomyopathy. Circ Res 2019; 124(9): 1360-1371. 


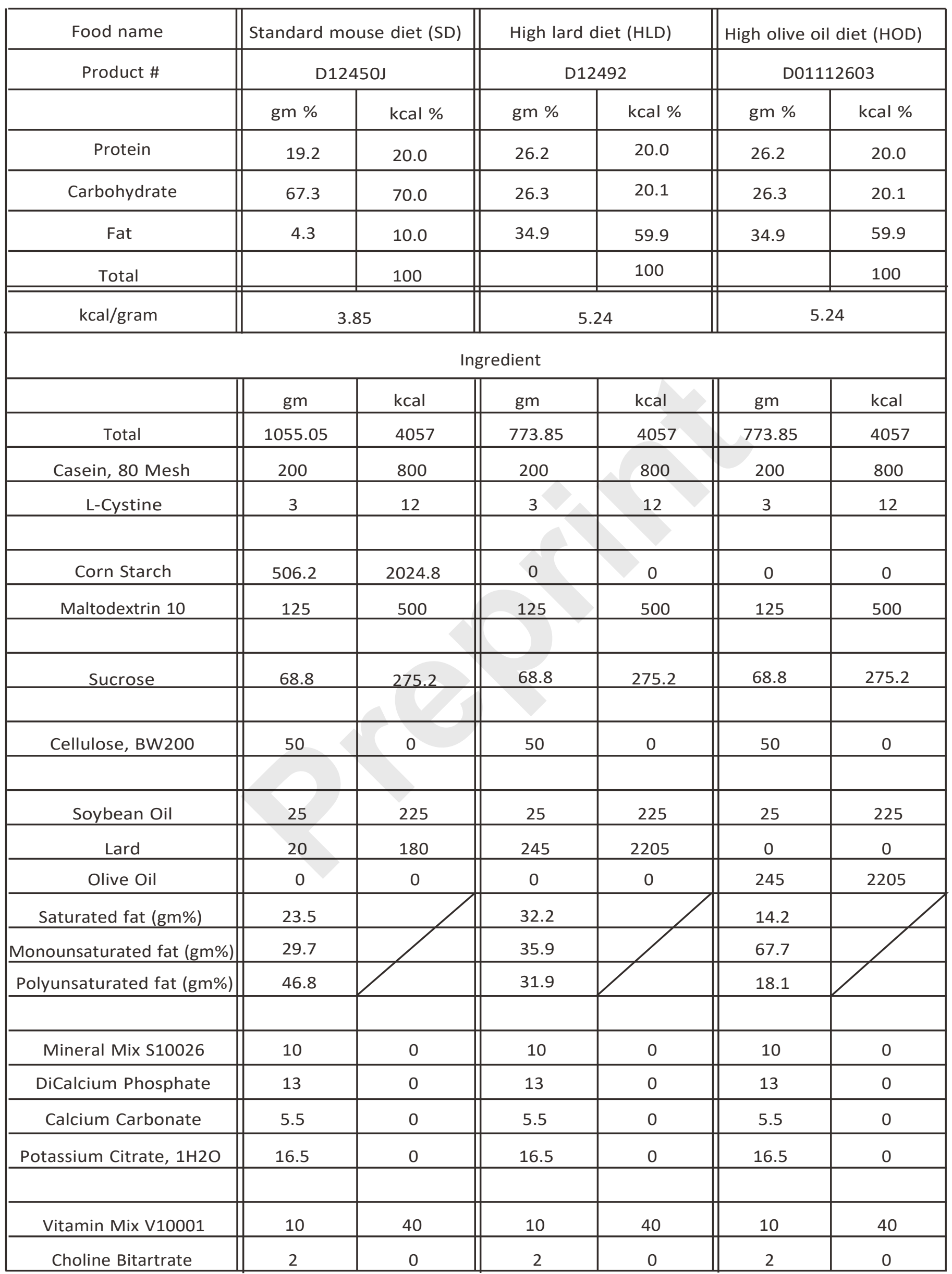




\section{Online Table 2}

\begin{tabular}{|c|c|c|}
\hline \multicolumn{3}{|c|}{ Primer sequences } \\
\hline Target gene & Forward ( 5' $\left.-3^{\prime}\right)$ & Reverse ( 5' $\left.-3^{\prime}\right)$ \\
\hline Gapdh & TCAACGGCACAGTCAAGG & CACGACATACTCAGCACC \\
\hline Myh7 & CCTGCGGAAGTCTGAGAAGG & CTCGGGACACGATCTTGGC \\
\hline Tgfb1 & CCACCTGCAAGACCATCGAC & CTGGCGAGCCTTAGTTTGGAC \\
\hline Col1a1 & GCTCCTCTTAGGGGCCACT & ATTGGGGACCCTTAGGCCAT \\
\hline Cpt1b & GACTTCCGGCTTAGTCGGG & GAATAAGGCGTTTCTCCAGGA \\
\hline Cd36 & ATGGGCTGTGATCGGAACTG & TTTGCCACGTCATTCTGGGTTT \\
\hline Acsl1 & TGCCAGAGCTGATTGACATTC & GGCATACCAGAAGGTGGTGAG \\
\hline Sod2 & AGACCTGCCTTACGACTATGG & CTCGGTGGCGTTGAGATTGTT \\
\hline Catalase & GGAGGCGGGAACCCAATAG & GTGTGCCATCTCGTCAGTGAA \\
\hline Srebf1 & TGACCCGGCTATTCCGTGA & CTGGGCTGAGCAATACAGTTC \\
\hline Fas & AGGTGGTGATAGCCGGTATGT & TGGGTAATCCATAGAGCCCAG \\
\hline Abca1 & AACAACCCCTGCTTCCGTTAT & GGCGAGACACGATGGACTTG \\
\hline Scd1 & TTCTTGCGATACACTCTGGTGC & CGGGATTGAATGTTCTTGTCGT \\
\hline Ddit3 & AAGCCTGGTATGAGGTCGC & TTCCTGGGGATGAGATAGGTG \\
\hline Hspa5 & ACTTGGGGACCACCTATTCCT & GTTGCCCTGATCGTTGGCTA \\
\hline spliced Xbp1 & TGAGAACCAGGAGTTAAG & CCTGCACCTGCTGCGGAC \\
\hline Dnajb9 & TCAGAGCGACAAATCAAAAAGGC & CTATTGGCATCCGAGAGTGTTT \\
\hline Atf4 & CCTGAACAGCGAAGTGTTGG & TGGAGAACCCATGAGGTTTCAA \\
\hline Tfam & AACACCCAGATGCAAAACTTTC & GACTTGGAGTTAGCTGCTCTTT \\
\hline Ppara & GGAAGACCACTCGCATTCCTT & GTAATCAGCAACCATTGGGTCA \\
\hline Pgc1a & TATGGAGTGACATAGAGTGTGC & GTCGCTACACCACTTCAATCC \\
\hline Nrf1 & AGCACGGAGTGACCCAAAC & AGGATGTCCGAGTCATCATAAG \\
\hline Pnpla2 & ATGTTCCCGAGGGAGACCAA & GAGGCTCCGTAGATGTGAGTG \\
\hline
\end{tabular}




\begin{tabular}{|c||l|l|}
\hline Dgat1 & GTGCCATCGTCTGCAAGATTC & GCATCACCACACACCAATTCAG \\
\hline Trpc1 & GATGTGCTTGGGAGAAATGCT & ACTGACAACCGTAGTCCAAAAG \\
\hline Trpc3 & GCCTTCATGTTCGGTGCTC & GGTCACCTCCAGATGCTCATT \\
\hline Trpc6 & GGCGGCTCTCTAAAGGCTG & TGGGGTAGTAGCCATACGGTG \\
\hline
\end{tabular}




\section{Online Table 3.}

\section{Comparison of cardiac function}

\begin{tabular}{|l|l|l|l|}
\hline mice & Wild-type & Wild-type & Wild-type \\
\hline Food type & SD & HLD & HOD switch \\
\hline N & 10 & 10 & 10 \\
\hline Heart Rate (beats $/ \mathrm{min})$ & $501 \pm 5$ & $505 \pm 6$ & $503 \pm 4$ \\
\hline CO $(\mathrm{ml} / \mathrm{min})$ & $21 \pm 2$ & $20 \pm 3$ & $19 \pm 4$ \\
\hline ESP $(\mathrm{mmHg})$ & $94 \pm 3$ & $111 \pm 4^{*}$ & $113 \pm 2{ }^{*}$ \\
\hline EDP $(\mathrm{mmHg})$ & $5.0 \pm 0.5$ & $10.4 \pm 0.4{ }^{*}$ & $7.2 \pm 0.3^{*} \#$ \\
\hline ESPVR $(\times 100 \mathrm{mmHg} / \mu \mathrm{L})$ & $202 \pm 24$ & $205 \pm 24$ & $203 \pm 25$ \\
\hline EDPVR $(\times 100 \mathrm{mmHg} / \mu \mathrm{L})$ & $3.3 \pm 0.2$ & $7.5 \pm 0.4 *$ & $5.1 \pm 0.3^{*} \#$ \\
\hline IVS $(\times 10 \mathrm{~mm})$ & $8.0 \pm 0.2$ & $9.7 \pm 0.2^{*}$ & $8.5 \pm 0.2{ }^{*} \#$ \\
\hline PW $(\times 10 \mathrm{~mm})$ & $7.9 \pm 0.3$ & $9.5 \pm 0.3^{*}$ & $8.4 \pm 0.2{ }^{*} \#$ \\
\hline LVEDD $(\times 10 \mathrm{~mm})$ & $35.6 \pm 0.4$ & $38.2 \pm 0.4 *$ & $37.0 \pm 0.3^{*} \#$ \\
\hline LVESD $(\times 10 \mathrm{~mm})$ & $20.2 \pm 0.3$ & $21.8 \pm 0.4$ & $21.1 \pm 0.5$ \\
\hline FS $(\%)$ & $42 \pm 1$ & $43 \pm 3$ & $43 \pm 2$ \\
\hline EF $(\%)$ & $70 \pm 3$ & $69 \pm 3$ & $69 \pm 4$ \\
\hline
\end{tabular}

Abbreviations: CO, cardiac output; ESP, end-systolic pressure; EDP, end-diastolic pressure; ESPVR, end-systolic pressure-volume relationship; EDPVR, end-diastolic pressure-volume relationship; EF, ejection fraction; FS, fractinal shortening; IVS, interventricular septum thickness during diastole; LV, left ventricular; LVEDD, left ventricular enddiastolic diameter; LVESD, left ventricular end-diastolic diameter; PW, posterior wall thickness during diastole; ${ }^{*} P<0.05$ vs $\mathrm{SD}, \# P<0.05$ vs HLD. Data show means $\pm \mathrm{SE}$. 


\section{Online Table 4.}

Comparison of cardiac function

\begin{tabular}{|c|c|c|c|}
\hline mice & SIRT1CKO & SIRT1CKO & SIRT1CKO \\
\hline Food type & SD & HLD & HOD switch \\
\hline $\mathrm{N}$ & 10 & 10 & 10 \\
\hline Heart Rate (beats/min) & $502 \pm 4$ & $502 \pm 5$ & $503 \pm 7$ \\
\hline $\mathrm{CO}(\mathrm{ml} / \mathrm{min})$ & $20 \pm 3$ & $20 \pm 4$ & $21 \pm 3$ \\
\hline $\mathrm{ESP}(\mathrm{mmHg})$ & $95 \pm 2$ & $113 \pm 4^{*}$ & $112 \pm 3$ * \\
\hline $\mathrm{EDP}(\mathrm{mmHg})$ & $5.1 \pm 0.3$ & $15.3 \pm 0.3^{*}$ & $10.2 \pm 0.3^{*} \#$ \\
\hline $\operatorname{ESPVR}(\times 100 \mathrm{mmHg} / \mu \mathrm{L})$ & $201 \pm 25$ & $200 \pm 26$ & $207 \pm 22$ \\
\hline $\operatorname{EDPVR}(\times 100 \mathrm{mmHg} / \mu \mathrm{L})$ & $2.8 \pm 0.6$ & $11.6 \pm 0.3^{*}$ & $8.1 \pm 0.2 * \#$ \\
\hline IVS (×10 mm) & $7.9 \pm 0.3$ & $12.9 \pm 0.3$ * & $9.9 \pm 0.2{ }^{*} \#$ \\
\hline $\mathrm{PW}(\times 10 \mathrm{~mm})$ & $7.8 \pm 0.3$ & $11.9 \pm 0.1$ * & $9.7 \pm 0.2{ }^{*} \#$ \\
\hline LVEDD (×10 mm) & $35.5 \pm 0.5$ & $39.3 \pm 0.3$ * & $37.2 \pm 0.2 * \#$ \\
\hline LVESD $(\times 10 \mathrm{~mm})$ & $20.2 \pm 0.4$ & $21.2 \pm 0.6$ & $21.1 \pm 0.5$ \\
\hline FS (\%) & $43 \pm 2$ & $45 \pm 3$ & $43 \pm 2$ \\
\hline $\mathrm{EF}(\%)$ & $69 \pm 4$ & $71 \pm 3$ & $69 \pm 4$ \\
\hline
\end{tabular}

Abbreviations: CO, cardiac output; ESP, end-systolic pressure; EDP, end-diastolic pressure; ESPVR, end-systolic pressure-volume relationship; EDPVR, end-diastolic pressure-volume relationship; EF, ejection fraction; FS, fractinal shortening; IVS, interventricular septum thickness during diastole; LV, left ventricular; LVEDD, left ventricular enddiastolic diameter; LVESD, left ventricular end-diastolic diameter; PW, posterior wall thickness during diastole; ${ }^{*} P<0.05$ vs $\mathrm{SD}, \# P<0.05$ vs HLD. Data show means $\pm \mathrm{SE}$. 


\section{Figure 1}

A

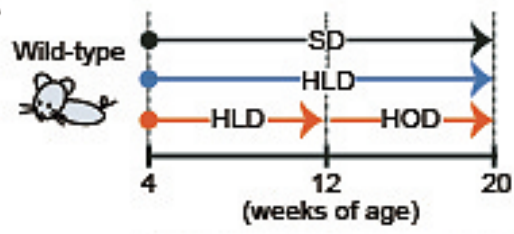

口SD $\mathrm{BHLD}$ 口HOD switch
Standard mouse diet (SD)

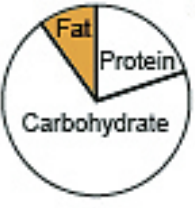

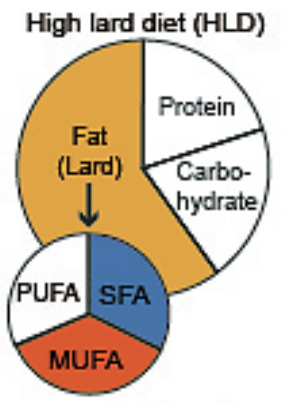

High olive oil diet (HOD)

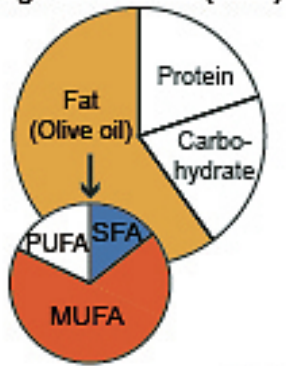

E
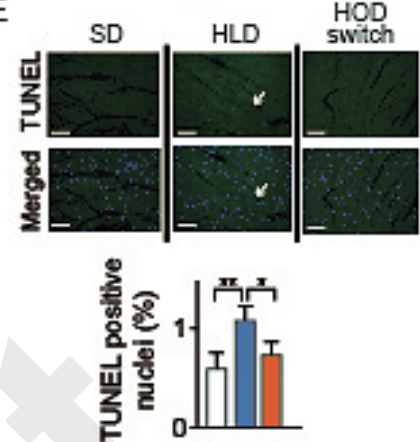

H

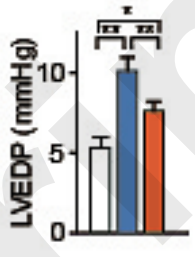

G

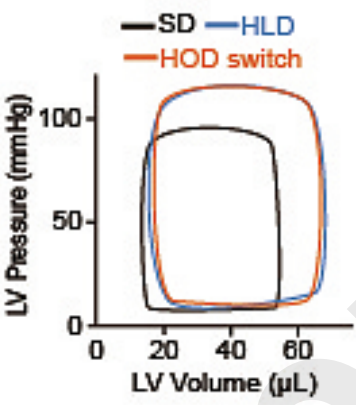

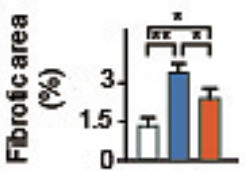

Figure 1. Switching from a high-lard diet to a high olive oil diet reduces high-lard diet-induced cardiomyopathy. (A) Protocol for the high-fat diet and composition of the diet. Wild-type mice were fed a standard mouse diet (SD) or high-lard diet (HLD) for 16 weeks or fed a HLD for 8 weeks and then switched to a high olive oil diet (HOD) for 8 weeks (HOD switch). (B) Heart sections stained with hematoxylin \& eosin (top): heart weight (HW) normalized by tibia length (TL; bottom). Scale bars: $1 \mathrm{~mm}$. ( $\mathrm{n}=$ 6). (C) Heart sections stained with wheat germ agglutin (top): cross-sectional cell surface area of cardiomyocytes (bottom). ( $n=3$ ). Scale bars: $100 \mu \mathrm{m}$. (D) Heart sections stained with Azan blue (top); fibrotic area (bottom). ( $n=3$ ). Scale bars: $100 \mu \mathrm{m}$. (E) Heart sections stained with terminal deoxynucleotidyl transferase dUTP nick end labeling (TUNEL): TUNEL-positive cardiomyocytes (arrows; top and bottom). ( $n=3$ ). Scale bars: $200 \mu \mathrm{m}$. (F) Relative expression of Myh7, Tgfb1, and Col1a1 in hearts. ( $(n=6)$. (G) Representative pressure-volume curve (left); left ventricle end-diastolic pressure (LVEDP; right). ( $n=10)$. (H) End-diastolic pressure volume relationship (EDPVR). $(n=10)$. Data are shown as mean (SEM). ${ }^{x} P<0.05,{ }^{x x} P<0.01$; analysis of variance followed by a post hoc Tukey-Kramer test.

Main Figure 1 


\section{Figure 2}
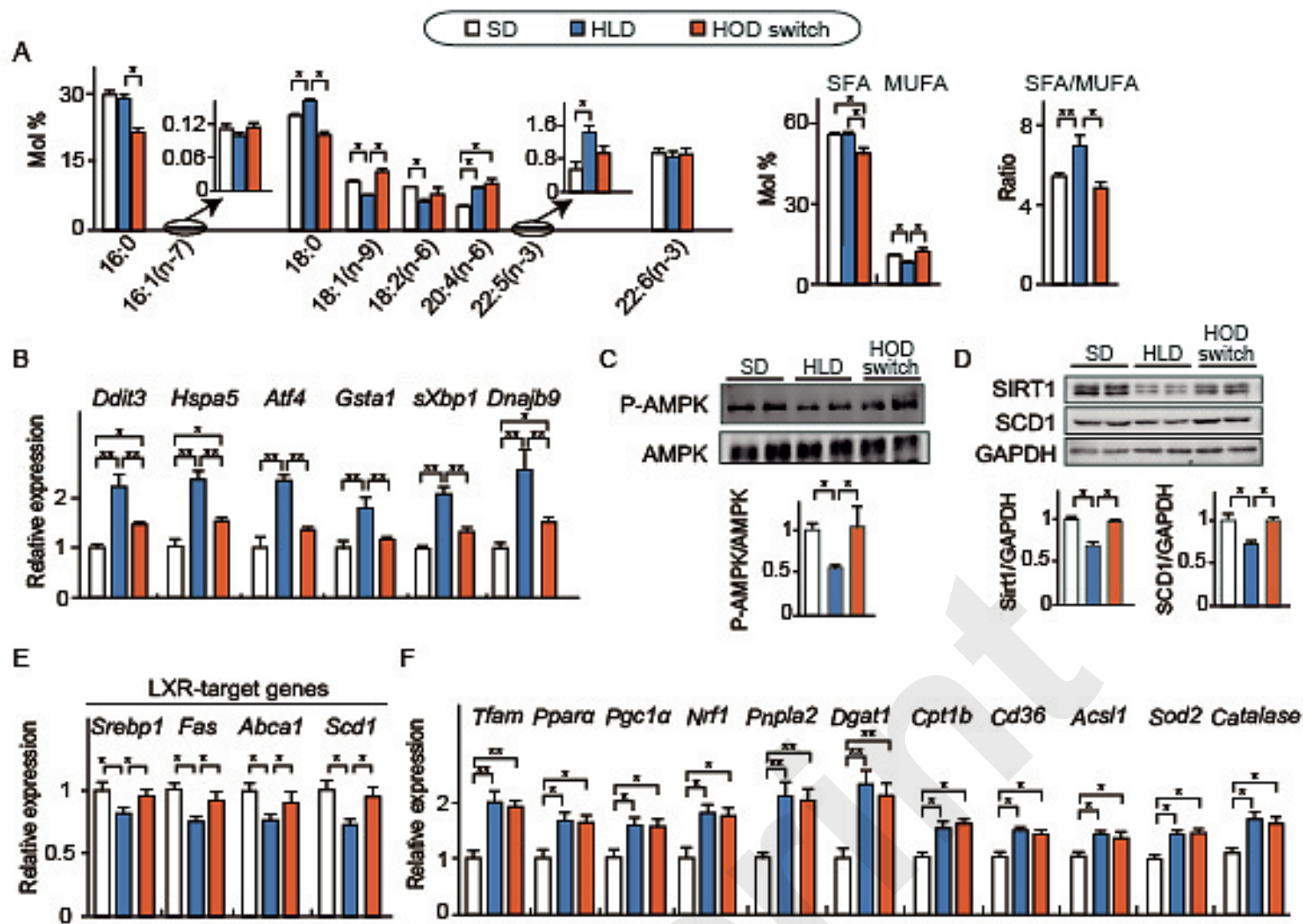

F

Figure 2. Increase in membrane ratio of saturated fatty acid to monounsaturated fatty acid and endoplasmic reticulum stress induced by a high-lard diet were reduced by switching to a high olive oil diet. (A) Saturated fatty acids (SFA).

monounsaturated fatty acids (MUFA), and polyunsaturated fatty acids in heart membrane (mol\% of phospholipids; left and middle); ratio of SFA to MUFA (right). $(n=4)$. (B) Relative expression in the heart of genes related to endoplasmic reticulum stress. ( $n=6)$. (C)-(D) Immunoblots of phosphorylated adenosine monophosphate-activated protein kinase (Thr172; P-AMPK), SIRT1, stearoyl-CoA desaturase-1 (SCD1), and glyceraldehyde 3-phosphate dehydrogenase (GAPDH) in hearts (top) and densitometry analyses (bottom). ( $n=4$ ). (E) Relative expression in hearts of genes targeting liver $X$ receptors. ( $n=6)$. (F) Relative expression of mitochondrial biogenesis, triglyceride turnover, peroxisome proliferator-activated receptor alpha (PPARa)-target, and forkhead box protein 01 (Fox01)-target genes in hearts. $\left(n=6\right.$ ). Data are shown as mean (SEM). ${ }^{x} P<0.05$, ${ }^{\mathrm{x}} P<0.01$; analysis of variance followed by a post hoc Tukey-Kramer test.

Main Figure 2 
Figure 3

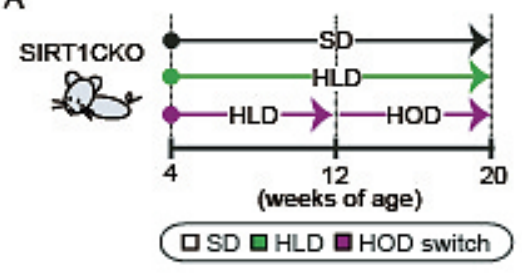

D

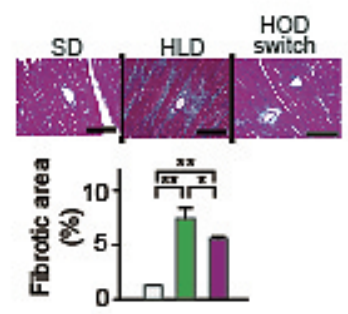

E
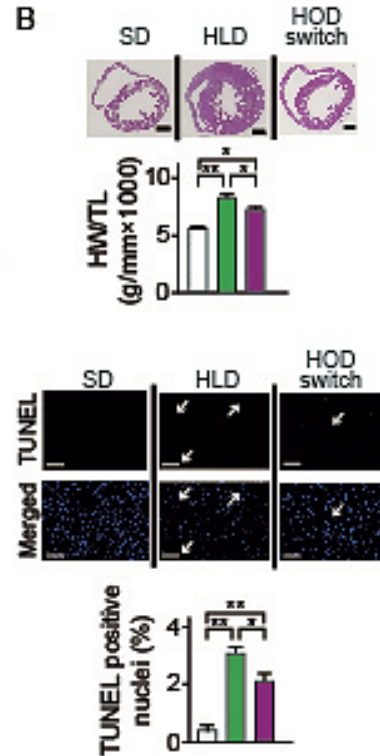

C
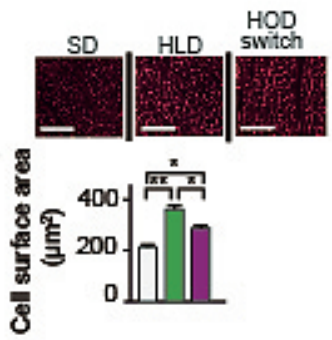

F

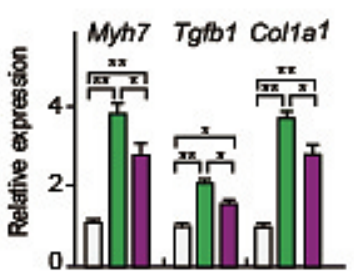

G
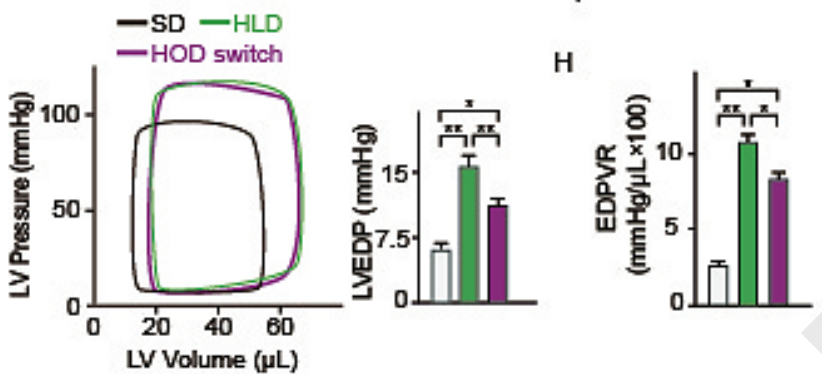

Figure 3. In SIRT1 knockout mice, switching to a high olive oil diet has a protective effect against cardiomyopathy induced by a high-lard diet. (A) Protocol for the high-fat diet. Cardiomyocyte-specific SIRT1 knockout mice (SIRT1CKO) were fed a standard mouse diet (SD) or high-lard diet (HLD) for 16 weeks or fed a HLD for 8 weeks and then switched to a high olive oil diet (HOD) for 8 weeks (HOD switch). (B) Heart sections stained with hematoxylin \& eosin (top); heart weight (HW) normalized by tibia length (TL; bottom). Scale bars: $1 \mathrm{~mm}$. $(n=6)$. (C) Heart sections stained with wheat germ agglutin (top): cross-sectional cell surface area of cardiomyocytes (bottom). ( $n=3$ ). Scale bars: $100 \mu \mathrm{m}$. (D) Heart sections stained with Azan blue (top); fibrotic area (bottom). ( $n=3$ ). Scale bars: $100 \mu \mathrm{m}$. (E) Heart sections stained with terminal deoxynucleotidyl transferase dUTP nick end labeling (TUNEL): TUNEL-positive cardiomyocytes (arrows; top and bottom). ( $n=3$ ). Scale bars: $200 \mu m$. (F) Relative expression of $M y h 7$. Tgfb1, and Colfa1 in hearts. ( $n=6$ ). (G) Representative pressure-volume curve (left). Left ventricle end-diastolic pressure (LVEDP: right). $(n=10)$. $(H)$ End-diastolic pressure volume relationship (EDPVR). $(n=10)$. Data are shown as mean $(S E M) .{ }^{x} P<0.05,{ }^{x x} P$ $<0.01$; analysis of variance followed by a post hoc Tukey-Kramer test.

Main Figure 3 
Figure 4

A 口SD $\square$ GLD $\square$ HOD switch
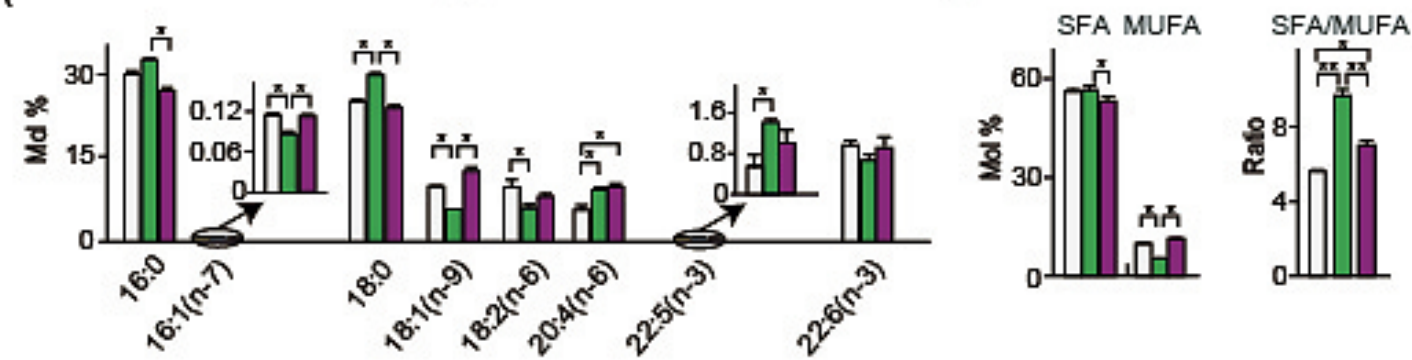

B

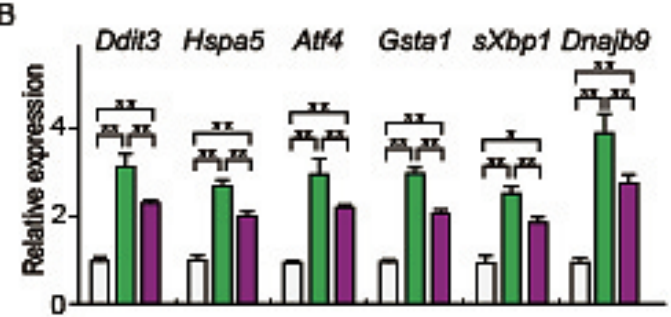

C

E
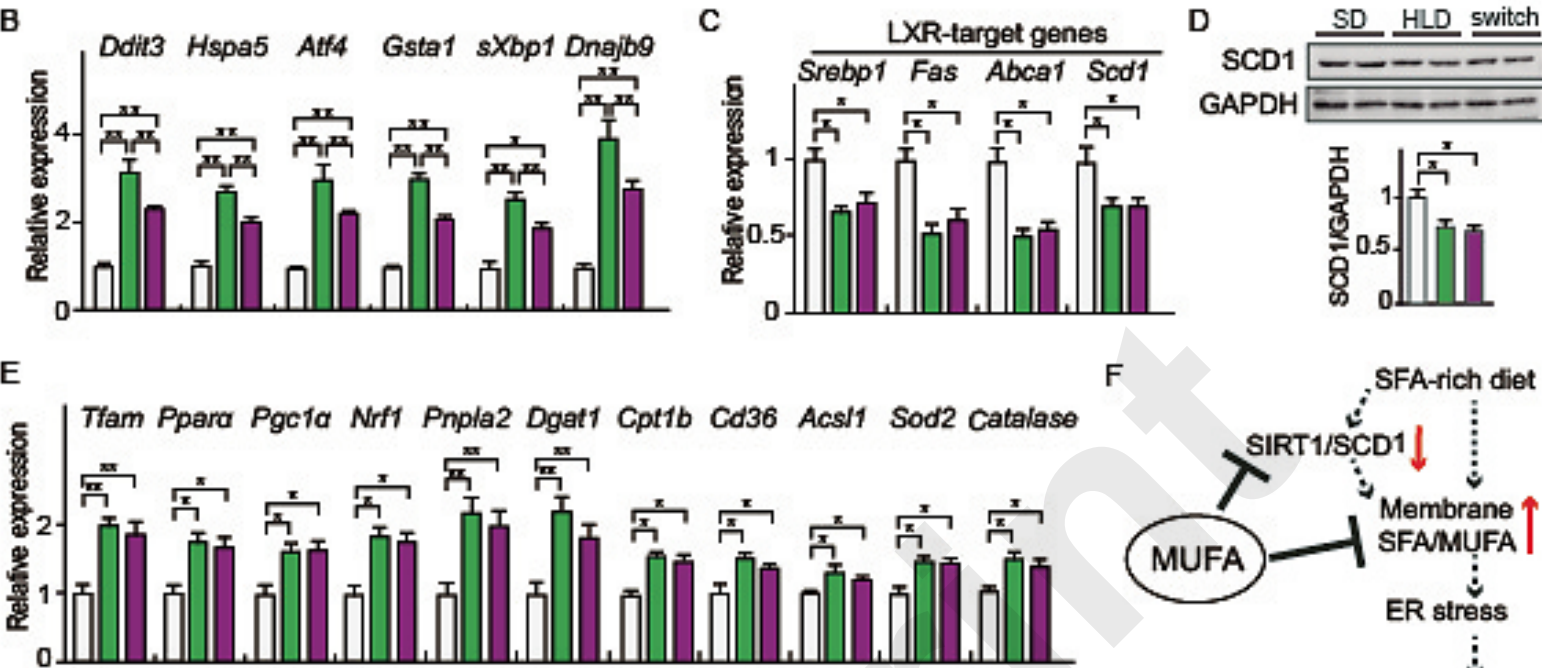

F

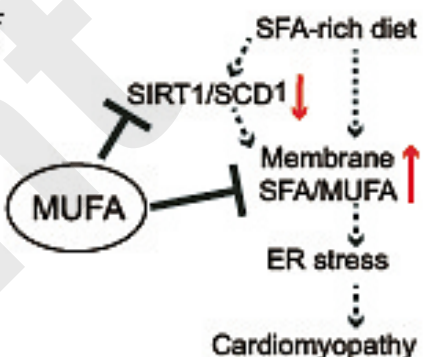

Figure 4. In SIRT1 knockout mice, increase in membrane ratio of saturated fatty acid to monounsaturated fatty acid and endoplasmic reticulum stress induced by a high-lard diet were reduced by switching to a high olive oil diet. (A)

Saturated fatty acids (SFA), monounsaturated fatty acids (MUFA), and polyunsaturated fatty acids in heart membrane (mol\% of phospholipids; left and middle); ratio of SFA to MUFA (right). ( $n=4)$. (B) Relative expression in the heart of genes related to endoplasmic reticulum stress. ( $n=6$ ). (C) Relative gene expression in hearts of genes targeting liver $X$ receptors. ( $n=6$ ). (D) Immunoblots of SCD1 and GAPDH in hearts (top): densitometry analysis (bottom). ( $n=4)$. (E) Relative expression in hearts of mitochondrial biogenesis, triglyceride turnover, PPARa-target, and FoxO1-target genes. ( $n=6)$. (F) Proposed working model. Data are shown as mean (SEM). ${ }^{x} P<0.05,{ }^{x x} P<0.01$; analysis of variance followed by a post hoc Tukey-Kramer test.

Main Figure 4 


\section{Online Figure 1}

\section{口SD QHLD QHOD switch}

A

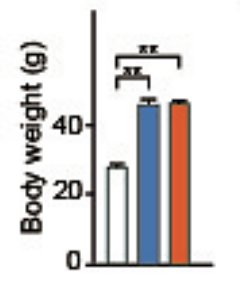

B

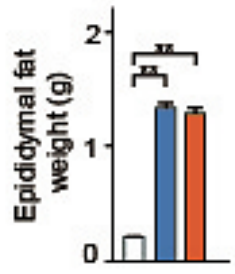

$\mathrm{C}$ - SD HLD HOD switch
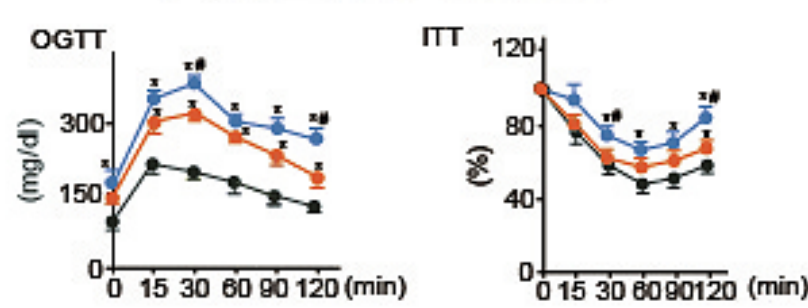

D

E

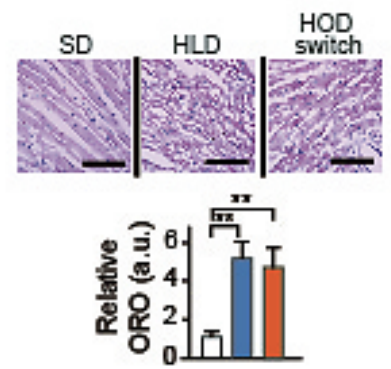

$\mathbf{F}$

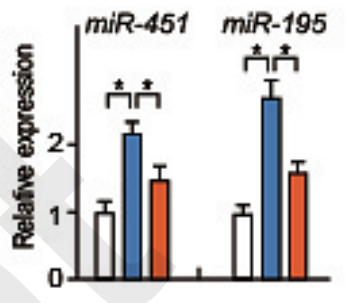

Online Figure 1. Metabolic impacts in wild-type mice fed either high lard diet or high olive oil diet switching. (A) Body weight. $(n=10)$. (B) Epididymal fat weight. $(n=6)$. (C) Oral glucose tolerance test (OGTT) and insulin tolerance test (ITT). ( $n=5$ ). (D) Relative gene expression of TRPC channels in hearts. $(n=6)$. (E) Heart sections stained with Oil red O (ORO; top); relative ORO area (bottom). ( $n=3$ ). Scale bars: $100 \mu \mathrm{m}$. (F) Relative microRNA (miR) expression in hearts. ( $n=6)$. Data were presented as mean \pm SEM. ${ }^{x} P<0.05,{ }^{n x} P<0.01$,by ANOVA followed by post hoc Tukey-Kramer test. 


\section{Online Figure 2}

A

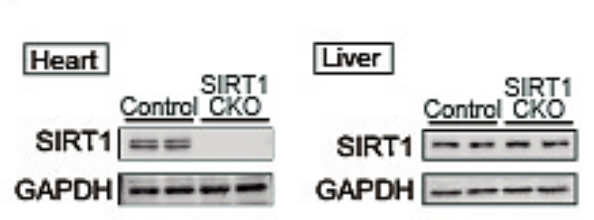

B

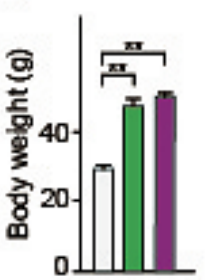

(एSD UHLD QHOD switch

C

D

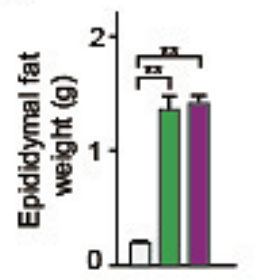

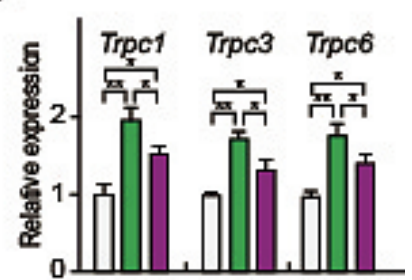

Online Figure 2. Metabolic impacts in cardiomyocyte-specific SIRT1 knockout mice fed either high lard diet or high olive oil diet switching. (A) Immunoblots for Sirt1 and GAPDH in hearts and livers. (B) Body weight. $(n=10)$. (C) Epididymal fat weight. ( $n=6$ ). (D) Relative gene expression of TRPC channels in hearts. $(n=6)$. Data were presented as mean \pm SEM. ${ }^{x} P<$ $0.05,{ }^{x x} P<0.01$,by ANOVA followed by post hoc Tukey-Kramer test. 